\title{
Influence of Snack Intake on Cardiac Autonomic Nervous System in Patients with Type 2 Diabetes
}

\author{
Saman Parvaneh ${ }^{1,2}$, Amir Abdolahi $^{2}$, Mehrnoosh Arafati ${ }^{1}$, Faeze Naderi ${ }^{1}$ \\ ${ }^{1}$ Islamic Azad University, Science and Research Branch, Tehran, Iran \\ ${ }^{2}$ Philips Research North America, Cambridge, MA, USA
}

\begin{abstract}
Food intake induces significant changes in heart rate $(H R)$ and heart rate variability (HRV) among healthy individuals. It is known that the cardiac autonomic nervous system (CANS) is impaired in patients with type 2 diabetes. The main goal of this pilot study was to investigate whether snack intake can affect CANS in this patient population. For this purpose, ECG was recorded pre- and post-snack intake in 15 volunteers with type 2 diabetes. Mean HR and short-term HRV measures (e.g., $S D N N, R M S S D$, percentage of power in $L F$ and $H F$ band, and $L F / H F$ ) were extracted and compared using the Wilcoxon signed-rank test between pre- and post-snack intake. From pre- to post-snack intake, mean glucose level increased by 24.1 points $(p=0.10)$ while mean heart rate increased by 1.9 beats per minute $(p=0.30)$. Changes in SDNN, RMSSD, power in LF band (\%), power in $H F$ band $(\%)$, and $L F / H F$ were not existent $(p=0.39,0.80$, $0.45,0.98,0.36$, respectively). As expected, an increase in glucose count was observed shortly after snack intake. No cardiac neural response, however, was observed in our sample of type 2 diabetics.
\end{abstract}

\section{Introduction}

Food intake induces significant changes to heart rate (HR) [1] and heart rate variability (HRV) among healthy individuals [2]. While cardiac neural response is impaired in type 1 diabetics who exhibit inconsistent patterns of heart rate variability (HRV) after eating [3], less is known about the influence of snack intake on cardiac neural response in individuals with type 2 diabetes. Furthermore, it is known that the cardiac autonomic nervous system (CANS) is impaired in patients with type 2 diabetes and low HRV is a risk factor for sudden cardiac death in this group [4, 5]. We hypothesized that snack intake can negatively affect CANS in this population group. Therefore, the objective of this study was to evaluate CANS response in type 2 diabetics after consuming a light snack.

\section{Data and Method}

This study is a secondary analysis of a retrospective data that was recorded in 2012.

\subsection{Data and Study Protocol}

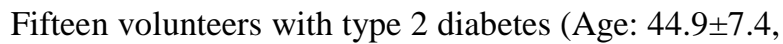
BMI: 27.4 $\pm 4.6, \quad 14 \%$ male) with no history of cardiovascular disease participated in this study. After signing a consent form, blood glucose level and electrocardiography (ECG) data were collected pre- and post-snack intake. ECG was recorded using the Novin s1800 monitoring device (Pooyandegan Rah Saadat Corporation, Tehran-Iran) and blood glucose level was measured using Glucosure Star (ApexBio, Taiwan).

ECG for baseline condition (pre-snack intake) was recorded for 3 minutes and then blood glucose was measured at the end of the baseline measurement. Participants were then asked to consume a light snack and after 15 minutes post-snack intake, ECG was recorded for a duration of 6 minutes. Blood glucose was measured for a second time at the end of the post-snack intake measurement. During the measurements, all participants were seated on a chair. The whole recorded ECG during the pre-snack intake ( 3 minutes) and the first 3 minutes of ECG recording during the post-snack intake were used for HR and HRV analysis. The study protocol is visualized in Figure 1.

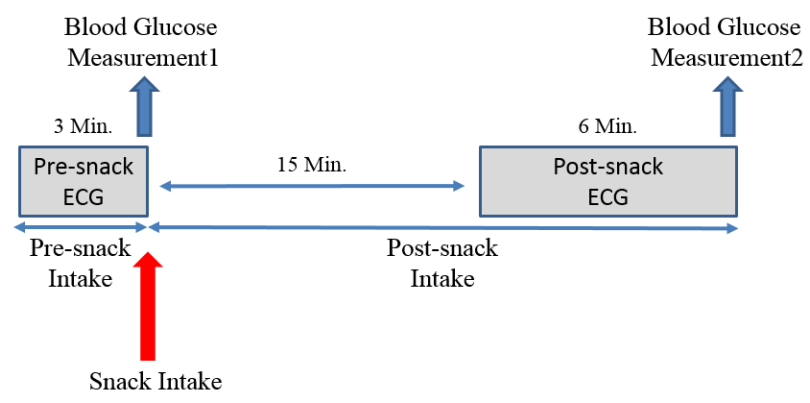

Figure 1. Study protocol 


\subsection{Method}

\subsubsection{QRS Detection}

Baseline wander was corrected using a moving average filter [6] and then Pan-Tompkins algorithms were used for QRS detection [7]. Detected QRS peaks were verified by manual inspection and were corrected if it was required. Using corrected QRS peaks, inter-beat (RR) interval series were created for HR and HRV analysis.

\subsubsection{Heart Rate and HRV}

RR intervals were used for estimation of different HR and HRV features for 3 minute recordings pre- and postsnack intake. The Kubios HRV Software was used for HRV analysis [8]. HR (minimum, maximum, and mean) and the following HRV measures were calculated in this study:

- Time-domain measures: standard deviation of RR intervals (SDNN), root mean square of successive differences (RMSSD), the relative number of successive intervals differing more than $50 \mathrm{~ms}$ (pNN50).

- Frequency-domain measures: percentage of power in low frequency (LF) band and high frequency (HF) band as well as LF/HF. HRV spectrum was estimated with FFT based Welch's periodogram method.

- Poincare plot measures: axis of ellipse fitted to points' distribution in Poincare plot (SD1 and SD2).

- Nonlinear measures: approximate and sample entropy.

Please refer to [8-10] for details on calculation of above-mentioned parameters.

\subsubsection{Statistical Analysis}

Means and standard deviations for continuous variables are reported. Pre- and post-test parameters were compared and tested using the Wilcoxon signed-rank test [11] at the significance level of 0.05 . For comparison of mean differences between pre- and post-snack intake, cohen's d effect size was calculated; values of $0.2,0.5$, and 0.8 were considered as small, moderate, and large effect sizes, respectively [12]. SAS version 9.3 was used for all statistical analysis (SAS Institute, Inc., Cary, NC).

\section{Results}

Means and standard deviations of HR and HRV measures pre- and post-snack intake are reported in Table 1. The obtained $p$-values are shown in Table 1 for extracted parameters. From pre- to post-snack intake, mean glucose level increased by 24.1 points $(\mathrm{p}=0.10)$ while mean heart rate increased slightly by 1.9 beats per minute $(\mathrm{p}=0.30)$. Changes in SDNN, RMSSD, power in LF band (\%), power in HF band (\%), and LF/HF were not existent $(\mathrm{p}=0.39,0.80,0.45,0.98,0.36$, respectively). Approximate entropy and sample entropy also did not change significantly from pre- to post-snack intake ( $\mathrm{p}=0.40$ and 0.14 , respectively), although increasing trends were noted. Cohen's d effect size for extracted parameters is reported in Table 2. Except for SD2 that had a moderate effect size $(d=0.52)$, all other parameters had small to almost moderate effect sizes.

Table 1. Means and standard deviations of HR and HRV measures pre- and post-snack intake along with p-value for paired comparison. $\downarrow$ and $\uparrow$ shows the decreasing and increasing trend, respectively.

\begin{tabular}{lccc} 
& $\begin{array}{c}\text { Pre-snack } \\
\text { intake }\end{array}$ & $\begin{array}{c}\text { Post-snack } \\
\text { intake }\end{array}$ & $P$-value \\
\hline $\begin{array}{l}\text { Blood Glucose, } \\
{[\mathrm{mg} / \mathrm{dl}]}\end{array}$ & $174.07 \pm 45.90$ & $198.13 \pm 52.06$ & $0.10 \uparrow$ \\
\hline Min HR, [bpm] & $71.73 \pm 9.26$ & $72.87 \pm 12.51$ & $0.57 \uparrow$ \\
\hline Max HR, [bpm] & $90.27 \pm 15.07$ & $90.00 \pm 12.45$ & $0.83 \downarrow$ \\
\hline Mean HR, [bpm] & $77.98 \pm 9.26$ & $79.92 \pm 10.62$ & $0.30 \uparrow$ \\
\hline SDNN, [ms] & $32.52 \pm 20.31$ & $24.74 \pm 11.74$ & $0.39 \downarrow$ \\
\hline RMSSD, [ms] & $22.17 \pm 14.32$ & $20.02 \pm 14.61$ & 0.80 \\
\hline pNN50, [\%] & $3.51 \pm 5.70$ & $5.02 \pm 9.49$ & $0.96 \uparrow$ \\
\hline pLF, [\%] & $32.53 \pm 15.67$ & $36.40 \pm 10.20$ & $0.45 \uparrow$ \\
\hline pHF, [\%] & $19.93 \pm 11.46$ & $19.07 \pm 12.87$ & $0.98 \downarrow$ \\
\hline LF/HF & $2.31 \pm 1.85$ & $3.12 \pm 2.33$ & $0.36 \uparrow$ \\
\hline SD1, [ms] & $15.71 \pm 10.15$ & $14.19 \pm 10.35$ & $0.78 \downarrow$ \\
\hline SD2, [ms] & $42.91 \pm 27.30$ & $31.58 \pm 13.82$ & $0.21 \downarrow$ \\
\hline Approximate & $0.93 \pm 0.16$ & $0.97 \pm 0.12$ & $0.40 \uparrow$ \\
Entropy & $1.56 \pm 0.38$ & $1.73 \pm 0.35$ & $0.14 \uparrow$ \\
\hline Sample Entropy & Min: Minimum, Max: Maximum &
\end{tabular}

Table 2. Cohen's d (d) for pairwise comparison of extracted parameters for between group differences.

\begin{tabular}{lc}
\cline { 2 - 2 } & Cohen's d effect size \\
\hline Blood Glucose, [mg/dl] & 0.49 \\
\hline Minimum HR, [bpm] & 0.10 \\
\hline Maximum HR, [bpm] & 0.02 \\
\hline Mean HR, [bpm] & 0.19 \\
\hline SDNN, [ms] & 0.47 \\
\hline RMSSD, [ms] & 0.15 \\
\hline pNN50, [\%] & 0.19 \\
\hline pLF, [\%] & 0.29 \\
\hline pHF, [\%] & 0.07 \\
\hline LF/HF & 0.39 \\
\hline SD1, [ms] & 0.22 \\
\hline SD2, [ms] & 0.52 \\
\hline Approximate Entropy & 0.28 \\
\hline Sample Entropy & 0.47 \\
\hline
\end{tabular}

\section{Conclusion}

In this article, blood glucose, heart rate, and heart rate variability for patients with type 2 Diabetes were compared pre- and post-snack intake. As expected, an increase in glucose count was observed shortly after 
snack intake. No cardiac neural response, however, was observed in our sample of type 2 diabetics. Recognizing the limitation of a small sample size, our findings suggest that $\mathrm{HR}$ and $\mathrm{HRV}$ in type 2 diabetics are insensitive to eating a snack.

\section{Acknowledgements}

The authors would like to thank the Pooyandegan Rah Saadat Corporation for providing vital sign monitoring device during the study period.

\section{References}

[1] B. Sciot, B. Vandenberk, S. Huijghebaert, G. Goovaerts, S. Van Huffel, J. Ector, et al., "Influence of food intake on the QT and QT/RR relation," Journal of electrocardiology, vol. 49, pp. 720-727, 2016.

[2] I. Dionne, M. White, and A. Tremblay, "The reproducibility of power spectrum analysis of heart rate variability before and after a standardized meal," Physiology \& behavior, vol. 75, pp. 267-270, 2002.

[3] D. Cozzolino, R. Furlan, D. Gruosso, C. Di Maggio, E. Miraglia del Giudice, R. Torella, et al., "Effects of a mixed meal on hemodynamics and autonomic control of the heart in patients with type 1 diabetes," The Journal of Clinical Endocrinology \& Metabolism, vol. 95, pp. 194-200, 2010.

[4] S. A. Smith, "Reduced sinus arrhythmia in diabetic autonomic neuropathy: diagnostic value of an age-related normal range," Br Med J (Clin Res Ed), vol. 285, pp. 15991601, 1982.

[5] M. Kataoka, C. Ito, H. Sasaki, K. Yamane, and N. Kohno, "Low heart rate variability is a risk factor for sudden cardiac death in type 2 diabetes," Diabetes research and clinical practice, vol. 64, pp. 51-58, 2004.

[6] S. Parvaneh, M. R. Hashemi Golpayegani, M. Firoozabadi, and M. Haghjoo, "Predicting the spontaneous termination of atrial fibrillation based on Poincare section in the electrocardiogram phase space," Proceedings of the Institution of Mechanical Engineers, Part H: Journal of Engineering in Medicine, vol. 226, pp. 3-20, 2012.

[7] J. Pan and W. J. Tompkins, "A real-time QRS detection algorithm," Biomedical Engineering, IEEE Transactions on, pp. 230-236, 1985.

[8] M. P. Tarvainen, J.-P. Niskanen, J. A. Lipponen, P. O. Ranta-Aho, and P. A. Karjalainen, "Kubios HRV-heart rate variability analysis software," Computer methods and programs in biomedicine, vol. 113, pp. 210-220, 2014.

[9] D. Nunan, G. R. Sandercock, and D. A. Brodie, "A quantitative systematic review of normal values for short-term heart rate variability in healthy adults," Pacing and Clinical Electrophysiology, vol. 33, pp. 1407-1417, 2010.

[10] M. V. Kamath, M. Watanabe, and A. Upton, Heart rate variability $(H R V)$ signal analysis: clinical applications: CRC Press, 2012.

[11] R. Woolson, "Wilcoxon Signed-Rank Test," Wiley encyclopedia of clinical trials, 2008.

[12] J. Cohen, "Statistical power analysis for the behavioral sciences (revised ed.)," ed: New York: Academic Press, 1977.
Address for correspondence.

Saman Parvaneh

2 Canal Park, 3rd floor, Cambridge, MA 02141

parvaneh@ieee.org 
Page 4 